\title{
A Survay on Appendiceal Involvement in Ovarian Mucinous Tumors
}

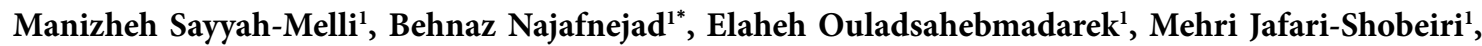 \\ Parvin Mostafa-Gharabaghi ${ }^{1}$
}

\begin{abstract}
Objectives: The ovarian mucinous tumors include $10 \%-15 \%$ of ovarian tumors. These tumors are either having ovarian origin or they are metastatic from other organs, particularly from gastrointestinal tract. According to ancient studies, routine appendectomy for tumours was determined based on differentiation of primary appendix carcinoma from ovarian tumors. However, recent investigations showed that appendectomy was performed only in cases with abnormal macroscopic appearance of appendix and in advanced stages of ovarian cancer. The aim of the present study is to investigate the correlation between macroscopic appearance of appendix and histopathology results of ovarian mucinous tumors as well as determining the stage of ovarian cancer appendices. Materials and Methods: This descriptive and cross-sectional study was conducted for inquiring about patients with pathologic diagnosis of ovarian and appendix mucinous tumors who had a surgery from 2010 to 2016 in Alzahra teaching hospital, Tabriz-Iran. The presented data were collected through a printed questionnaire followed by analysis.

Results: This study was done on 257 patients with definite diagnosis of ovarian and appendix mucinous tumors. Total number of 121 patients had undergone appendectomy and 110 cases of them had normal macroscopic appearance of appendix. Furthermore, $98.2 \%$ of patients with grossly normal looking appendix had normal pathology results and $63.6 \%$ of patients with abnormal looking appendix had abnormal pathology results. Totally $5.9 \%$ of patients with borderline and $30 \%$ of invasive ovarian mucinous tumors had affected appendix. A number of 4 patients were at stage III and 2 of the cases were at stage IV.

Conclusions: According to our investigation and previously published studies, appendectomy during ovarian tumor surgery is the only recommended option in cases with abnormal macroscopic appearance of appendix and advanced stages of ovarian mucinous cancer.

Keywords: Ovarian mucinous tumors, Appendix mucinous tumors, Ovarian cancer staging
\end{abstract}

\section{Introduction}

Ovarian mucinous tumors account for $10 \%$ to $15 \%$ of total gynecologic cancers which is a subset of epithelial ovarian neoplasms. Approximately $80 \%$ of primary ovarian cancers are classified as benign and the remainder evenly split between borderline and malignant (1-4), which $3 \%$ to $4 \%$ of them are mucinous carcinomas (3-5). The malignant tumors can be more commonly diagnosed at premenopausal period (i.e. ages between 40 to 50 years); meanwhile, they can be seen even at younger ages (6). These tumors may primarily have ovarian origin or may be metastatic with their origins in other parts of the body, particularly from gastrointestinal tract. The appendix is the source of $8 \%$ of ovarian mucinous tumors (7). The primary size of ovarian mucinous carcinomas is in the range of about 8 to $20 \mathrm{~cm}$ but they can be larger than this, too (5). They are usually cystic or solid, unilateral and restricted to ovary. The outer layer of the ovary is commonly smooth and without tumoral involvement (3). Meanwhile, the metastatic type is usually bilateral, and smaller in size with outer layer involvement (8-10). Pseudomyxoma peritonei can occur in association with ovarian mucinous carcinoma.
Although it is very rare, the origin of pseudomyxoma peritonei may be mucinous neoplasms originating from ovarian teratoma and rupture of an ovarian capsule containing primary ovarian mucinous tumor $(6,11,12)$. There exist various clinical challenges as it is difficult to diagnose and differentiate between primary ovarian mucinous and metastatic tumors. According to previous studies, routine appendectomy during ovarian mucinous cancer staging was suggested; it helps in differentiating primary appendiceal adenocarcinoma or gastrointestinal tract origin with ovarian metastases from primary ovarian mucinous tumor, particularly in borderline and malignant types $(1,13)$. Despite these facts, various investigations have shown that appendectomy should be performed only in appendices with abnormal macroscopic appearance. In patients with the normal macroscopic appearance of appendix or with borderline and malignant types of ovarian mucinous tumors at early stages, the possibility of appendiceal tumoral involvement is very rare and there is no need for routine appendectomy during staging of ovarian mucinous neoplasms $(1,10)$. The aims of the present study were three-fold: first, to measure the prevalence rate of 
concurrent appendiceal tumoral involvement in women with ovarian mucinous neoplasm; second, to assess whether abnormal macroscopic appearance of appendix is predictive of appendiceal pathologic involvement; and finally, to evaluate the correlation between type of ovarian mucinous neoplasm and the stage of cancer with appendiceal tumoral involvement.

\section{Materials and Methods}

This retrospective, observational, cross-sectional study was conducted by reviewing the records of patients with final diagnosis for benign, borderline and malignant ovarian and appendiceal mucinous tumors, admitted to and operated in department of obstetrics and gynecology of Alzahra hospital (Tabriz University of Medical Sciences) from March 2010 to September 2016. The research was done on database by searching the expressions "ovarian mucinous tumor" and "appendix carcinoma". Patients with all histologic types of ovarian and appendiceal mucinous neoplasms based on pathology records were included and patients with the history of bilateral oophorectomy or previous appendectomy were excluded from the study. Data were collected by the questionnaire, containing the type of ovarian mucinous tumor, type of appendix mucinous tumor, gross appearance of the appendix, and clinical staging based on operative report. Then, frequency, ratio and percentage were calculated based on given variables and chi-square statistical test was performed for determination of association between variables. $P$ value $<0.05$ was considered to be statistically significant.

\section{Results}

In this study, 257 patients who, based on postoperative final pathology, had ovarian and appendix mucinous neoplasm were surveyed. Two hundred eleven (82.1\%) patients had benign, 23 (8.9\%) had borderline, and 23 (8.9\%) had malignant mucinous tumors (Figure 1). Of 257 patients, 121 cases underwent appendectomy concurrent with ovarian surgery, 84 of these patients $(39.8 \%)$ had benign, 17 cases $(73.9 \%)$ had borderline, and 20 cases $(87 \%)$ had malignant ovarian mucinous tumors. Based on microscopic results, 9 cases, out of 121 appendectomy cases, had appendiceal mucinous tumoral involvement. Out of 9 patients, 2 patients had malignant appendiceal mucinous tumor without ovarian involvement and underwent surgery with primary diagnosis of pseudomyxoma peritonei, 1 patient had borderline and 6 patients had invasive ovarian mucinous tumors. Based on pathology results, in a survey of appendiceal macroscopic appearance and histopathology results, among patients with grossly normal looking appendix, 98.2\% had normal pathology and only 2 cases $(1.8 \%)$ had appendiceal tumor. Among patients with grossly abnormal looking appendix, $63.6 \%$ had appendiceal tumor (Figure 2).

The Association Between Ovarian Cancer Staging and Appendiceal Tumor

Based on the pathology results and operation records, among the patients with borderline ovarian mucinous tumors, 11 cases $(64.7 \%)$ were at stage I, 5 cases $(29.4 \%)$ at stage II, and 1 case (5.9\%) at stage III. Of the patients with invasive ovarian mucinous tumors, 7 cases (35\%) were at stage I, 6 cases (30\%) at stage II, 5 cases (25\%) at stage III, and 2 cases $(10 \%)$ at stage IV. One patient of borderline group (stage III) and 6 patients of malignant group (4 at stage III and 2 at stage IV) had appendiceal involvement (Figure 1).

\section{Discussion}

Results of this study demonstrated that routine appendectomy during surgery for ovarian invasive mucinous tumors at early stage is not beneficial. From among 257 patients with ovarian mucinous tumors, 121 patients had appendectomy during operation for ovarian tumor. Two patients had primary appendiceal malignant mucinous tumor and concurrent ovarian mucinous cystadenoma; theyhad been candidated for surgerybecause

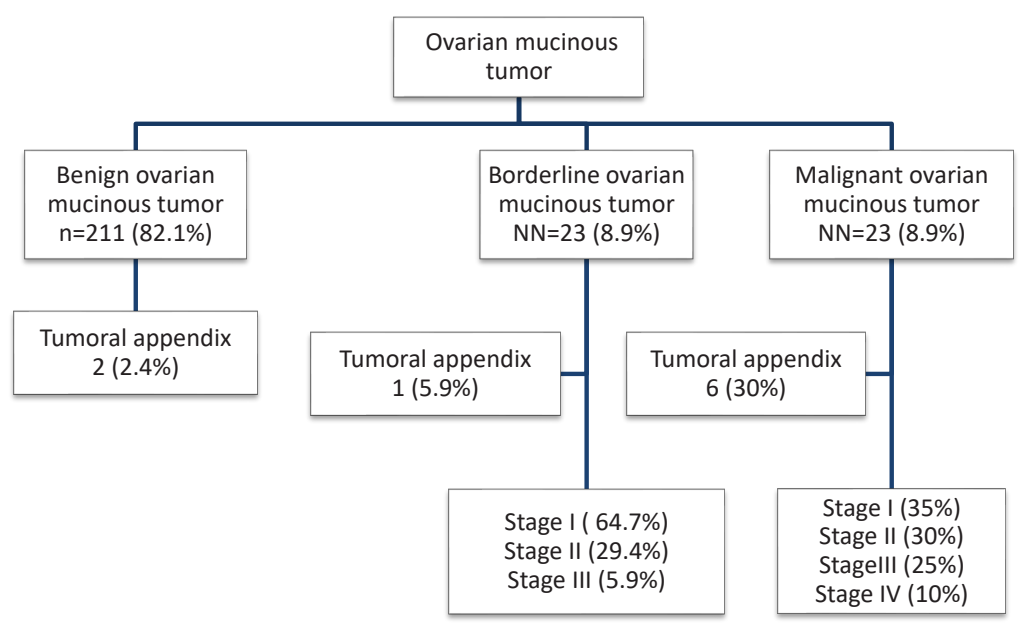

Figure 1. Results of the Study. 


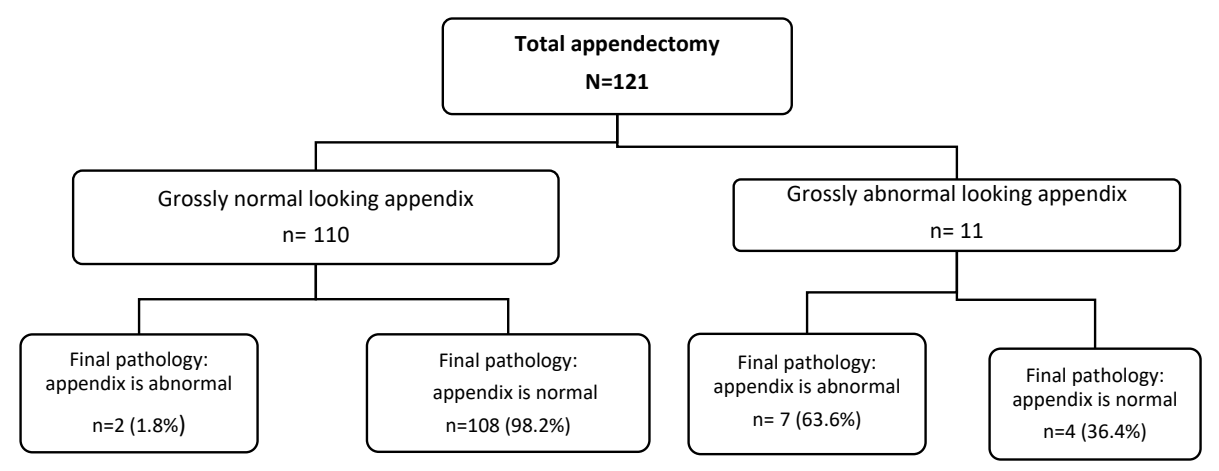

Figure 2. Appendectomy in Mucinous Tumor and Macroscopic and Final Pathology Results.

of pseudomyxoma peritonei, and in evaluation during the surgery, appendiceal malignant mucinous tumor was suggested based on appendiceal abnormal macroscopic appearance and metastatic disease (pseudomyxoma peritonei) without ovarian involvement. The remainder cases of appendiceal tumoral involvement had concurrent ovarian tumor so that, $5.9 \%$ of patients with borderline and $30 \%$ of patients with malignant tumors had metastatic appendiceal tumoral involvement. It seems that the extent of appendix involvement was related to the type of ovarian tumor and it was more common in malignant tumors $(P \leq 0.001)$. This is in line with the results of a previous study has been done by Moore et al who demonstrated that from among 45 cases with benign ovarian mucinous tumor, only 1 case had primary malignant appendix tumor (2). Furthermore, Timofeev et al reported $47 \%$ benign, $34 \%$ borderline and $19 \%$ invasive ovarian mucinous tumors among 76 patients with ovarian mucinous tumor and confirmed that appendix tumoral involvement was completely related to the type of ovarian tumor and 1 of 36 cases with benign, 1 of 26 cases with borderline and 1 of 14 cases with malignant ovarian mucinous had appendiceal metastasis (14). Lin et al (1), Westermann et al (15), and Malfetano et al (16) reported similar results. Based on the results of the present study, among patients with ovarian borderline mucinous tumor, only 1 case had malignant appendiceal tumoral involvement (stage III ovarian cancer). The stage of ovarian borderline tumor had not statistically significant correlation with the appendiceal tumoral involvement $(P=0.059)$ but the stage of ovarian malignant mucinous tumor had significant association with the possibility of appendiceal involvement $(P=0.0001)$. The higher the stage is, the more the possibility of appendiceal involvement would be, so that at stages III and IV the involvement would be the most $(P=0.0001)$. Currently, routine appendectomy during surgery at an early stage of ovarian cancer for rulling out the appendiceal involvement is a matter of controversy. Based on National Comprehensive Cancer Network Guidelines, appendectomy should be performed for all patients with ovarian mucinous tumor, regardless of the stage of disease for rulling out of the primary appendiceal cancer (17). Ramirez et al reported that among 57 patients with primary ovarian cancer undergoing appendectomy during primary surgery, 23 patients (40\%) with ovarian mucinous tumor had no appendiceal involvement (18). Similarly, in the study of Feigenberg et al on 453 patients undergoing ovarian mass surgery and appendectomy, it was reported that 77 patients were included in the study, from which 11 patients (14\%) with metastatic appendiceal carcinoma involved the ovary, 30 patients (39\%) with borderline, and 36 patients (47\%) with malignant ovarian mucinous tumor. Among these patients, 55 were at stage I and 11 patients were at stage III. Fifty-five patients had no pathologic evidence of appendiceal involvement by tumor, as authors could not establish documentation of necessary appendectomy at an early stage of tumor (10). The study of Fontanelli et al on 160 patients with ovarian mucinous tumor undergoing concurrent appendectomy showed that 120 patients were at stage I and stage II without pathologic evidence of appendiceal involvement (19). Malfetano et al reported similar results, it means, none of the 21 patients in an early stage of ovarian tumor had appendiceal malignant involvement (16).

Based on the results of the present study, the correlation between appendiceal macroscopic appearance and primary tumor of appendix or metastatic involvement of appendix was statistically significant $(P=0.0001)$. Normal appearance correlated with normal pathology (98.2\%) and only in $1.8 \%$ cases, despite normal appearance during surgery, appendices had malignant involvement in the pathology report. In abnormal looking appendix during surgery, in $63.6 \%$ of cases, there was pathologic evidence of primary malignant or metastatic involvement of appendix.

According to National Comprehensive Cancer Network guidelines, to prevent possible primary macroscpoic involvement of appendices, appendectomy should be performed in all definite ovarian mucinous tumors during surgery regardless of the appearance of appendices (17). Lin et al conducted a survey on 309 patients with ovarian tumor; 155 of patients underwent concurrent appendectomy and 56 of them had previous appendectomy history. From this population, 197 (64\%) were diagnosed 
with benign, 68 (22\%) with borderline, and 44 (16\%) with malignant ovarian mucinous tumors. Only 1 case with benign tumor had abnormal appendix appearance and definite pathology results. From among 112 patients with borderline and invasive ovarian mucinous tumor, 67 cases underwent concurrent appendectomy, and none of the patients with normal looking appendix had appendiceal malignant involvement (1). Feigenberg et al reported similar results, in that final pathology in all cases with normal looking appendix were negative (10). Moore et a1 also confirmed this result (2).

Through the present study, it can be inferred that in malignant ovarian tumors, appendix tumoral involvement had a significant correlation with abnormal appendix appearance $(P=0.059)$ but this issue could not be demonstrated for borderline and benign tumors $(P=0.071)$. Appendix tumoral involvement is independent of appendix macroscopic appearance and there is no correlation between appendix gross appearance and its tumoral involvement. These results differ from those of the previous few studies and were in contrast with Lin and colleagues' study which showed the dependence of gross appearance of appendix and its tumoral involvement on malignant and borderline tumors (1).

\section{Conclusions}

The results of this study can demonstrate that performing appendectomy during surgery for ovarian mucinous tumor would be prudent when patients are at an advanced stage of tumor, the macroscopic appearance of appendix is abnormal, and/ or there is evidence of the existence of a metastatic disease such as pseudomyxoma peritonei. In other situations, appendectomy is not a required procedure.

\section{Conflict of Interests}

Authors declare that they have no conflict of interests.

\section{Ethical Issues}

This study was approved by the Ethics Committee of Tabriz University of Medical Sciences under the number 1395.1303. Written informed consent was obtained from all participants in the study.

\section{Financial Support}

his study was funded by a grant from the Women's Reproductive Health Research Center, Tabriz University of Medical Sciences.

\section{Acknowledgements}

We express our sincere gratitude to Women's Reproductive Health Research Center, Tabriz University of Medical Sciences, and our honorable pathologists, Dr. Dastranj, Miss Balazade and Miss Ansari.

\section{References}

1. Lin JE, Seo S, Kushner DM, Rose SL. The role of appendectomy for mucinous ovarian neoplasms. Am J Obstet Gynecol. 2013;208(1):46.e41-44. doi:10.1016/j. ajog.2012.10.863

2. Moore L, Gajjar K, Jimenez-Linan M, Crawford R. Prevalence of Appendiceal Lesions in Appendicectomies Performed During Surgery for Mucinous Ovarian Tumors: A Retrospective Study. Int J Gynecol Cancer. 2016;26(8):1386-1389. doi:10.1097/igc.0000000000000800

3. Hart WR, Norris HJ. Borderline and malignant mucinous tumors of the ovary. Histologic criteria and clinical behavior. Cancer. 1973;31(5):1031-1045. doi:10.1002/1097-0142(197305)31:5<1031::AIDCNCR2820310501>3.0.CO;2-7

4. Hart WR. Mucinous tumors of the ovary: a review. Int J Gynecol Pathol. 2005;24(1):4-25.

5. Hoerl HD, Hart WR. Primary ovarian mucinous cystadenocarcinomas: a clinicopathologic study of 49 cases with long-term follow-up. Am J Surg Pathol. 1998;22(12):1449-1462.

6. Lee KR, Scully RE. Mucinous tumors of the ovary: a clinicopathologic study of 196 borderline tumors (of intestinal type) and carcinomas, including an evaluation of 11 cases with 'pseudomyxoma peritonei'. Am J Surg Pathol. 2000;24(11):1447-1464.

7. Riopel MA, Ronnett BM, Kurman RJ. Evaluation of diagnostic criteria and behavior of ovarian intestinaltype mucinous tumors: atypical proliferative (borderline) tumors and intraepithelial, microinvasive, invasive, and metastatic carcinomas. Am J Surg Pathol. 1999;23(6):617635. doi:10.1097/00000478-199906000-00001

8. Prayson RA, Hart WR, Petras RE. Pseudomyxoma peritonei. A clinicopathologic study of 19 cases with emphasis on site of origin and nature of associated ovarian tumors. Am J Surg Pathol. 1994;18(6):591-603.

9. Young RH, Gilks CB, Scully RE. Mucinous tumors of the appendix associated with mucinous tumors of the ovary and pseudomyxoma peritonei. A clinicopathological analysis of 22 cases supporting an origin in the appendix. Am J Surg Pathol. 1991;15(5):415-429.

10. Feigenberg $\mathrm{T}$, Covens $\mathrm{A}$, Ghorab $\mathrm{Z}$, et al. Is routine appendectomy at the time of primary surgery for mucinous ovarian neoplasms beneficial? Int J Gynecol Cancer. 2013;23(7):1205-1209. doi:10.1097/ IGC.0b013e31829b7dca

11. McKenney JK, Soslow RA, Longacre TA. Ovarian mature teratomas with mucinous epithelial neoplasms: morphologic heterogeneity and association with pseudomyxoma peritonei. Am J Surg Pathol. 2008;32(5):645-655. doi:10.1097/PAS.0b013e31815b486d

12. Ronnett BM, Seidman JD. Mucinous tumors arising in ovarian mature cystic teratomas: relationship to the clinical syndrome of pseudomyxoma peritonei. Am J Surg Pathol. 2003;27(5):650-657. doi:10.1097/00000478-20030500000008

13. Giri S, Lal P, Rawal S, Sekhon R. Routine appendicectomy be performed as a part of surgical staging in ovarian cancer. Int J Reprod Contracept Obstet Gynecol. 2016;5(12):3. doi:10.18203/2320-1770.ijrcog20164324 
14. Timofeev J, Galgano MT, Stoler MH, Lachance JA, Modesitt SC, Jazaeri AA. Appendiceal pathology at the time of oophorectomy for ovarian neoplasms. Obstet Gynecol. 2010;116(6):1348-1353. doi:10.1097/ AOG.0b013e3181fae628

15. Westermann C, Mann WJ, Chumas J, Rochelson B, Stone ML. Routine appendectomy in extensive gynecologic operations. Surg Gynecol Obstet. 1986;162(4):307-312.

16. Malfetano JH. The appendix and its metastatic potential in epithelial ovarian cancer. Obstet Gynecol. 1987;69(3 Pt 1):396-398.

17. Morgan RJ, Armstrong DK, Alvarez RD, et al. NCCN
Clinical Practice Guidelines in Oncology: Ovarian Cancer Including Fallopian Tube Cancer and Primary Peritoneal Cancer. 2016.

18. Ramirez PT, Slomovitz BM, McQuinn L, Levenback C, Coleman RL. Role of appendectomy at the time of primary surgery in patients with early-stage ovarian cancer. Gynecol Oncol. 2006;103(3):888-890. doi:10.1016/j. ygyno.2006.05.021

19. Fontanelli R, Paladini D, Raspagliesi F, di Re E. The role of appendectomy in surgical procedures for ovarian cancer. Gynecol Oncol. 1992;46(1):42-44. doi:10.1016/00908258(92)90193-M

(c) 2018 The Author (s); This is an open-access article distributed under the terms of the Creative Commons Attribution License (http://creativecommons.org/licenses/by/4.0), which permits unrestricted use, distribution, and reproduction in any medium, provided the original work is properly cited. 